\title{
Unusual Pc1 Geomagnetic Pulsations: Case study: 11 September 2017
}

\author{
F.Z. Feygin ${ }^{1}$, Yu.G. Khabazin ${ }^{1}$, N.G. Kleimenova ${ }^{1}$, T. Raita ${ }^{2}$, L.M. Malysheva ${ }^{1}$ \\ ${ }^{1}$ Schmidt Institute of the Physics of the Earth RAS, Moscow, Russia \\ ${ }^{2}$ Sodankylä Geophysical Observatory, Sodankylä, Finland \\ e-mail: feygin@ifz.ru, kleimen@ifz.ru, khabazin@ifz.ru, tero.raita@sgo.fi
}

Accepted: 2 August 2019

\begin{abstract}
The results of the analysis of the structure and dynamics of unusual Pc1 geomagnetic pulsations, recorded at the Finnish pulsation magnetometer chain in the end of 24-th solar activity cycle, are presented. The discussed event was observed on 11 September 2017 in the late recovery phase of the severe magnetic storm that occurred on 7-8 September 2017. The storm recovery phase is typical time for the Pc1 pulsation generation. However, in course of this storm, only one Pc1 event was recorded with a very unusual complicated dynamic spectrum which was found to be similar at all five ground-based stations, spaced from 57 to $67^{\circ}$ geomagnetic latitude. The Pc1 amplitude maximum has been observed at the lowest latitude station Nurmijarvi (NUR, $L=3.3$ ). The theoretical interpretation of the behavior of this unusual of Pc 1 pulsation burst is presented.
\end{abstract}

\section{(c) 2018 BBSCS RN SWS. All rights reserved}

Keywords: magnetosphere, geomagnetic activity, pulsations, Alfven waves, solar wind

\section{Introduction}

Geomagnetic pulsations Pc1, known as "pearls", are the most striking type of the electromagnetic ion cyclotron (EMIC) waves in the frequency band of 0.5-2.0 Hz. Experimental studies have shown that the ground-based Pc1 pulsations ("pearls") are observed on the form of the periodically successive Alfven wave packets with the predominantly left-hand wave polarization. These pulsations had been the subject of the intense research for many years (e.g., Matveeva and Troitskaya, 1965; Troitskaya and Guglielmi, 1969; Feygin and Yakimenko, 1969; Gendrin at al., 1971; Troitskaya et al., 1975; Kangas et al., 1998; Feygin et al., 2003; Mursula, 2007; Demekhov, 2007). The duration of the Pc1 series is usually from the half of an hour to the several hours. The Pc1 pulsations are the most frequently observed on the decline and in the minimum of the solar activity cycle (e.g., Matveeva and Troitskaya, 1965; Guglielmi et al., 2006; Mursula, 2007). It is well established that the Pc1 waves are generated via the cyclotron resonant interaction of waves and particles in the Earth's magnetosphere and propagate toward the Earth surface along the magnetic field lines. The plasmapause region could be the plausible region of the Pc1 Alfven wave generation (e.g., Troitskaya and Guglielmi, 1969, Feygin and Yakimenko, 1969; Gendrin at al., 1971; Kangas et al., 1998; Demekhov, 2007).

The generation of Pc1 pulsations is typical for the late recovery phase of a magnetic storm (e.g., Wentworth, 1964), when the ring current decay occurs, and the plasmasphere is filled with the cold plasma, which improves the conditions for the development of the cyclotron resonance which leads to the excitation of $\mathrm{Pc} 1$ pulsations.

In spite of a good understanding of the properties and generation mechanisms of these pulsations, their relation to other geomagnetic perturbations is still insufficiently investigated. This is especially true for the unusual Pc1 events, one of which we are considering here.

The aim of this paper is to analyze the Pcl geomagnetic pulsation event with a very unusual dynamic spectrum observed on 11 September 2017 in the end of the recovery phase of the strong magnetic storm on 7-8 September 2017. We used the ground-based data from the Finnish pulsation magnetometer profile consisting of five search coil magnetometers spaced from 57 to $67^{\circ}$ geomagnetic latitude. A reasonable interpretation scenario will be presented.

\section{Observation}

The severe two-steps magnetic storm with the lowest $\mathrm{SymH}$ index value about -150 nT had happened on 7-8 September 2017 in the end of the declining phase of the solar cycle 24. The progression of the planetary magnetic activity ( $\mathrm{Kp}$ index) as well as SymH index is shown in Fig. 1 for the first half of September 2017. It is seen that the storm recovery phase started after 8 September 2017, and the Kp index dropped up to $\mathrm{Kp}=0$ for about two days. Unfortunately, there were no OMNI data for these two days.

In course of this storm, only one Pc1 event was recorded at the Finnish pulsation coil magnetometer chain in the early morning (03-09 MLT) on 11 September 2017. To demonstrate the unusual behavior of this Pc1 event, we present here an example (6 March 2011) of the dynamic spectrum of the "classical" Pc1 waves (Fig. 2). It was well established that the typical dynamical spectra of the Pc1 pearl pulsations are characterized by a weak change in the carrier frequency which is seen in Fig. 2. However, the spectrum of the Pc1 event on 11 September 2017 discussed here demonstrates a very unusual complicated spectral feature presented in Fig. 3 which is significantly different from the typical Pc1 pearl pulsations spectra shown in Fig. 2.

The geographical map of the location of the Finnish pulsation magnetometer stations is shown in the left side of Fig. 3. The right side of Fig 3 displays the dynamic spectrum of the Pc1 event under consideration. The very unusual wave spectral structure, similar at all five pulsation stations, is seen. The maximum of the Pc1 amplitude was observed at the lowest latitude station Nurmijarvi (NUR, $L=3.3$ ). $L$ is the McIllwain parameter.

It is known that the most favourable area of Pc1 generation locates in the vicinity of the plasmapause. Due to that, we tried to consider the possible dynamic of the plasmapause location in course of the studied time interval. Unfortunately, we could not find the direct satellite measurements of the plasmapause in this day. Due to that, we used the model estimation.

The empirical model (Moldwin et al., 2002) provides the plasmapause location $(L p p)$ as $L p p=5.39-0.382 \mathrm{Kp}$, where Kp is the maximal $\mathrm{Kp}$ index value for previous 12 hours. We suppose that in the morning on 10 September, the plasmapause was located at $L \sim 5.4$ due to the previous 12 hours $\mathrm{Kp}$ index value was zero (Fig. 1). However, in the early morning of 11 st September, the plasmapause could shifted to $L=4.2$ because the previous 12 hours maximal $\mathrm{Kp}$ index value was $\sim 3$ (Fig. 1). 


\section{GFZ German Research Contre for Geosclonces \\ PLANETARY MAGNETIC THREE-HOUR-RANGE INDICES Kp, 2017}
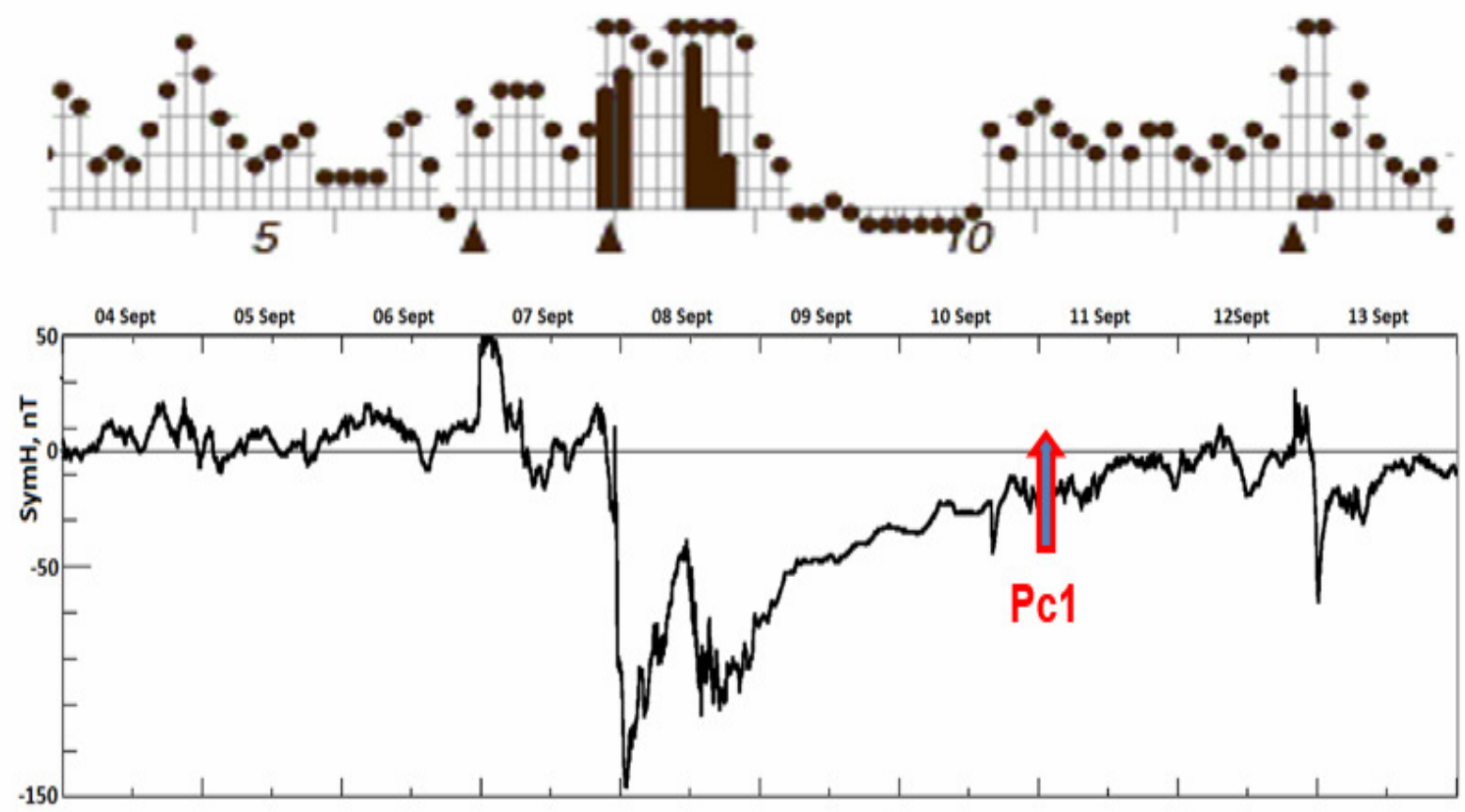

Fig. $1 \mathrm{Kp}$ and SymH variations on 4-13 September 2017.

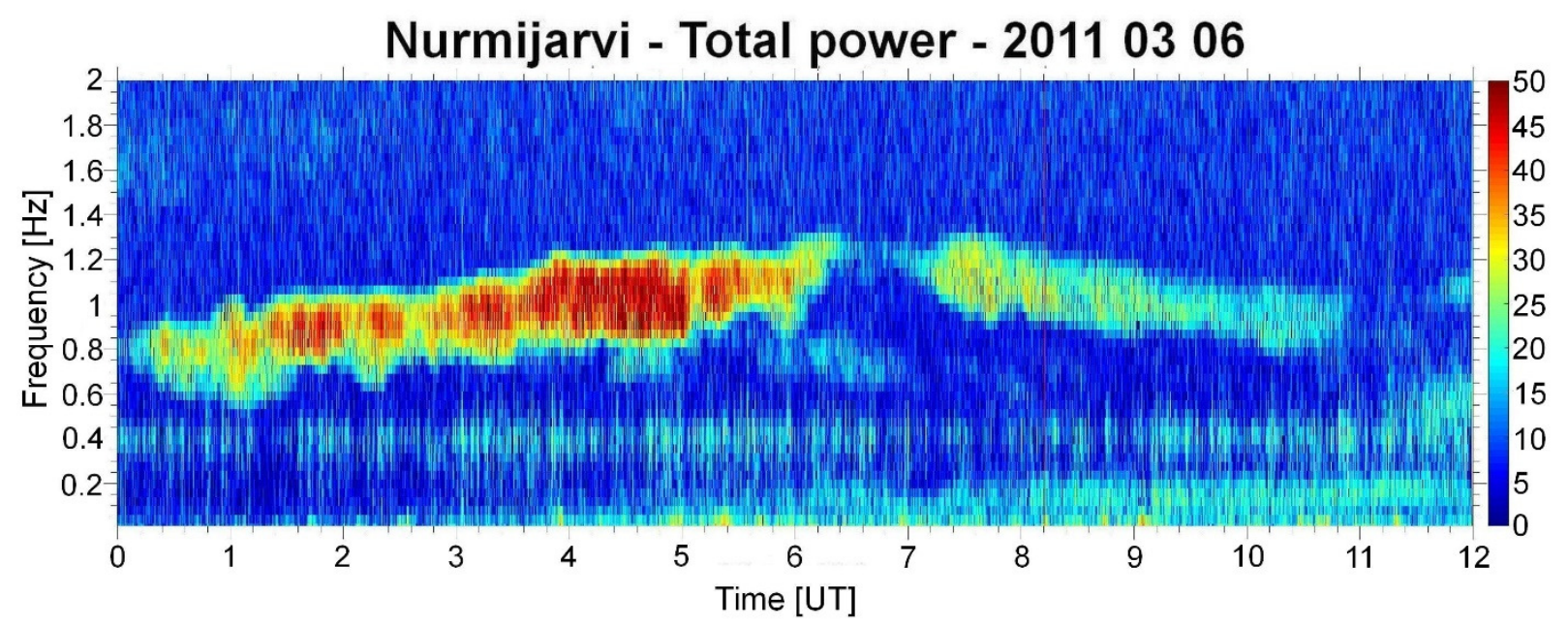

Fig. 2. Pc1 pulsations (total power) registered at Nurmijarvi station on 6 March 2011.

Figure 4 demonstrates the conditions in the Interplanetary Magnetic Field (IMF By and IMF Bz components) as well as solar wind velocity (V), dynamic pressure (Psw) and proton density $(\mathrm{Np})$ during the considered time interval of $\mathrm{Pc} 1$ pulsations. It is seen the significant decreasing of the Psw and $\mathrm{Np}$ values. We can expect that due to strong decrease of the solar wind pressure (Psw) from $6 \mathrm{nPa}$ to $2 \mathrm{nPa}$ (Fig. 4), the plasmasphere started to expand and the plasmapause shifted to the higher $L$-shells.

\section{Discussion}

The considered unusual Pc1 pulsation had a complex structure and could to represent the superposition of two different emissions generated simultaneously at two different source locations.

The higher frequency Pc1 emission event presents a series of bursts (frequency range 2-3 Hz) with a follow-up period from 7 to $15 \mathrm{~min}$. Each burst lasts about 20-25 min, in which the central frequency is practically unchanged. But every next burst starts at a lower frequency. All the bursts have a wide frequency spectrum $\Delta f / f_{0} \approx 0.4$ ( $f_{0}$ is the central frequency) compared to the classical "pearls" ( $\Delta f / f_{0} \approx 0.1$ ). The overall frequency trend is from $2.5 \mathrm{~Hz}$ to $1.5 \mathrm{~Hz}$ and continues from $01.00 \mathrm{UT}$ to $02.30 \mathrm{UT}$.

The nature of this Pc1 event could be explained by applying the generally accepted model of the resonant interaction of the electromagnetic ion cyclotron (EMIC) waves with hot anisotropic protons in the Earth's magnetosphere (e.g., Kangas et al., 1998).

Let us now consider parameters, which control the variation of the Pc1 central frequency. The latter corresponds to the maximum of the EMIC wave growth rate (e.g., Feygin and Yakimenko, 1971, Gendrin et al., 1971) given by

$$
f_{0} \approx f_{p} c_{A} / v_{T},
$$


where $f_{p}$ is the equatorial proton gyrofrequency, $c_{A}$ is the Alfven velocity and $v_{T}$ is the proton thermal velocity. According to Eq. (1) the central frequency depends on the $L$-shell value. To demonstrate this we note that $f_{p} \propto B$, and $c_{A} \propto B / \sqrt{n}$, where $\mathrm{n}$ is the plasma number density. For adiabatic motions, the thermal proton velocity scales change as $\sqrt{B}$.

On the other hand, the plasma number density varies in the dayside magnetosphere as $n \propto L^{-2}$ for a plasmasphere (Carpenter and Anderson, 1992). Assuming a dipole field geometry, the magnetic field intensity scales as $B \propto L^{-3}$. Using these scaling laws we obtain the following approximate variation for the central frequency with the $L$ :

$$
f_{0} \propto L^{-3.5}
$$

It is known that the most favourable area of Pc1 generation locates in the vicinity of the plasmapause. During the considered time interval, due to strong decrease of the solar wind pressure (Psw) from $6 \mathrm{nPa}$ to $2 \mathrm{nPa}$ (Fig. 4), the plasmasphere expanded and the plasmapause shifted to the higher $L$-shells.

According to that, we suppose that the source of Pc1 pulsation is shifted to higher $L$-shells as well in the recovery phase of the magnetic storm, i.e. to the regions with a reduced magnetic field and density of the background plasma. Thus, three parameters control the frequency of Pc1 pulsations (see Eqs. 1,2). Such motion of the source could lead to decrease of the frequency of Pc1, as it is seen at all considered stations at 01-03 UT (Fig. 3, 5).

The low-frequency part of the emission resembled the classical Pc1 pulsations lasting 4 hours at slightly increasing central frequency (from $\sim 1.4$ to $\sim 1.7 \mathrm{~Hz}$ ) but with unusually large bandwidth $(\sim 0.6 \mathrm{~Hz})$. Such Pc1 emissions usually are generated in vicinity of the plasmapause. We suppose that these wave generation associates with so called 'old' plasmapause corresponded to that which was formed during quiet period $(\mathrm{Kp}=0)$. The measured by Finnish statins, the distribution of the wave arriving direction (did not shown here) confirmed that the sources of the high and low frequency waves were different because these waves arrived from the different directions.

Another feature of the events under consideration is rather broad dynamic spectra of the emissions (Fig. 5a). Now consider parameters which control the dynamic spectra of the emissions. According to the results obtained by (Gendrin et al., 1971), the frequency width of the Pc1 spectrum is controlled by the magnetic field and density of the background plasma at the top of the geomagnetic field line, and we could roughly estimate that as

$$
\Delta f \propto B^{1 / 2} n^{3 / 2}
$$

$$
\Delta f \propto L^{-4.5}
$$

We suppose that to the morning on 11 September, the plasmapause could shifted to $L=4.2$ because the previous 12 hours maximal Kp index value was $~ 3$ (Fig. 1). That is the source could be located in a region with the increased magnetic field and density of the background plasma. According to $(3,4)$ the Pc1 spectral width was enhanced too. Later on due to strong decrease of the solar wind pressure (Fig. 4), the plasmasphere expanded and the plasmapause shifted to the higher $L$-shells, i.e. to the region of the lower magnetic field and lower density of the background plasma, the Pc1 spectrum width became reduced.

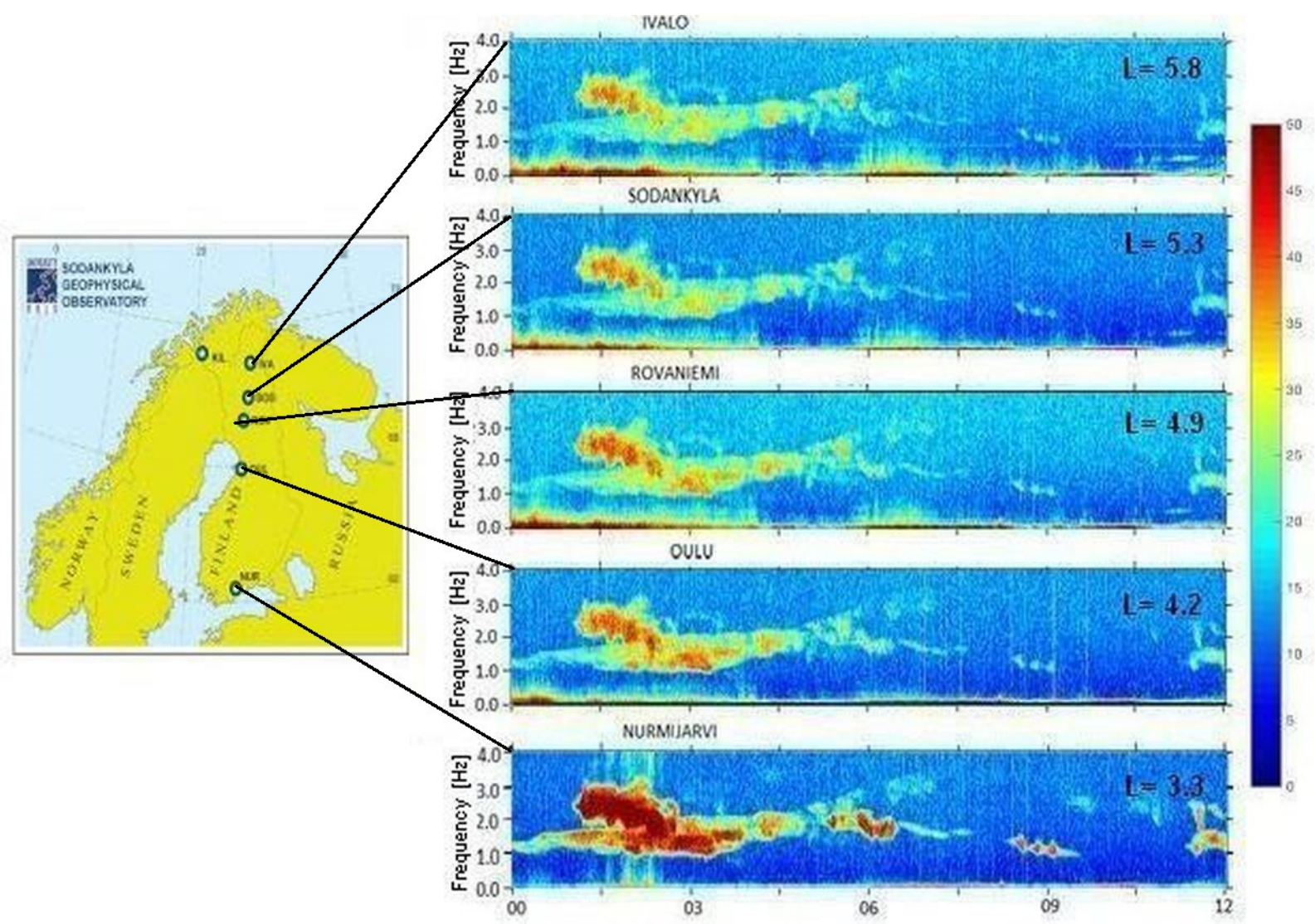

Fig. 3. Pc1 spectrograms (total power) at Scandinavian stations on 11September 2017. 


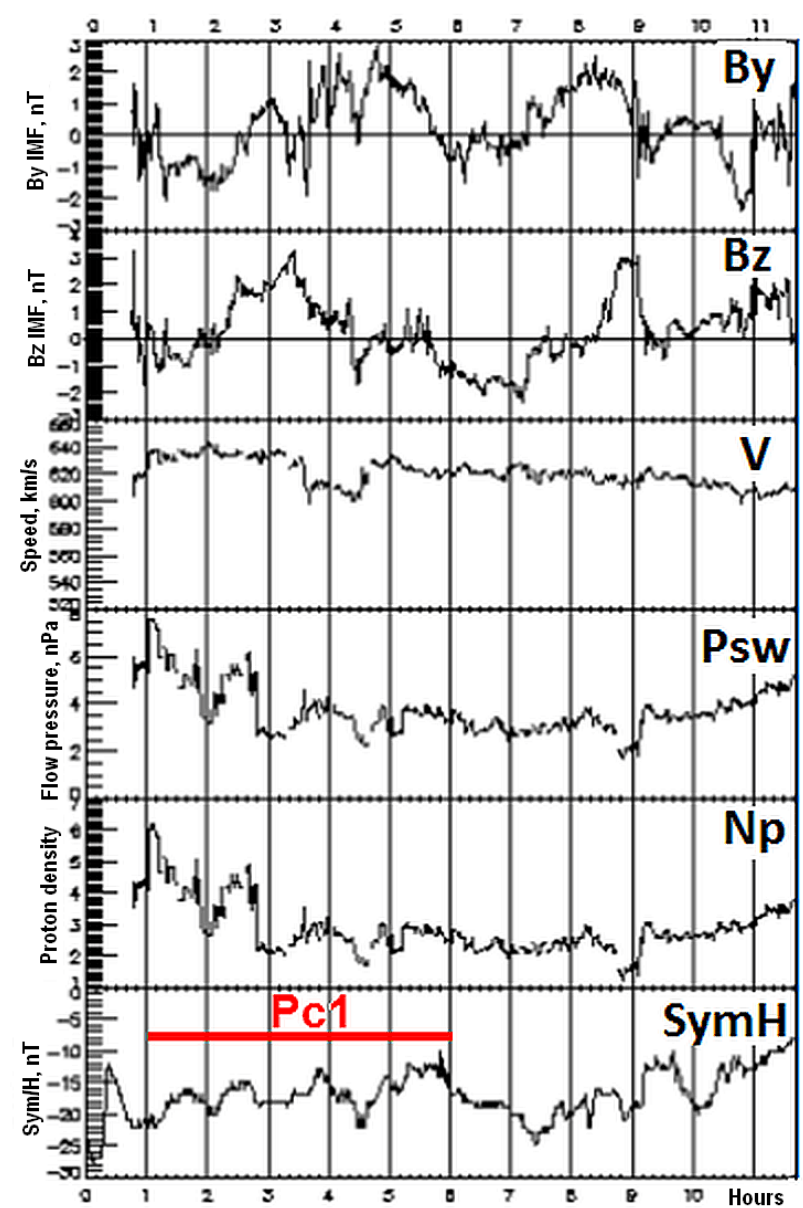

Fig. 4. The IMF and solar wind data on 11 September 2017 at 00-12 UT.

11.09.2017
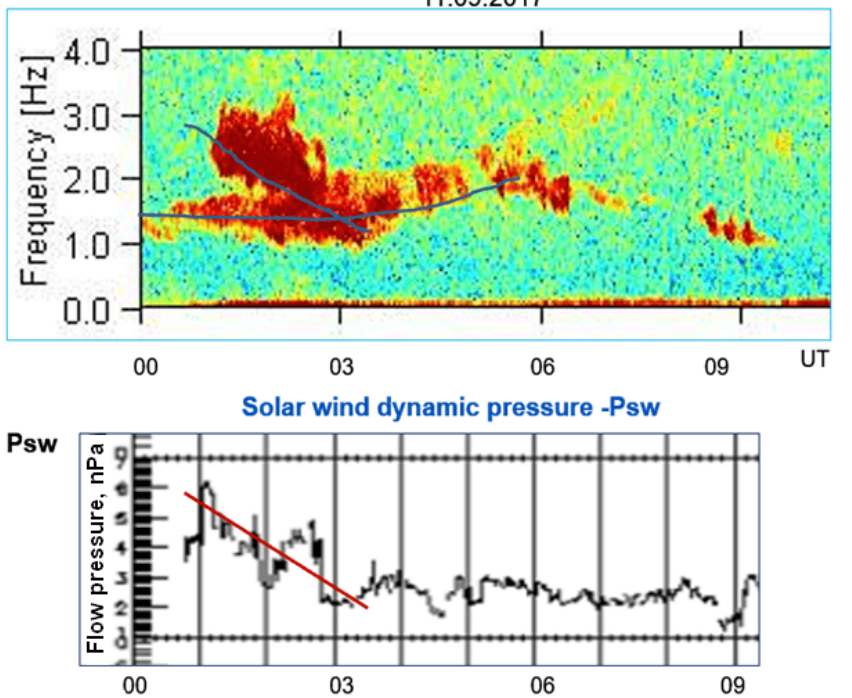

Fig. 5. The Pc1 spectrogram (H-component) at NUR (upper plot) and Psw variations (bottom plot) on 11 September 2017.
The rather broad dynamic spectra of the emissions could be also result of the spectral broadening in the process of the quasilinear interaction EMIC wave with energetic anisotropic protons. A quasilinear approximation had been fruitfully used in the theory of the geomagnetic pulsations (Feygin and Yakimenko, 1969; Gendrin at al., 1971; Feygin and Kurchashov 1975). Feygin and Kurchashov (1975) have done a numerical experiment allowing permanent control of the quasilinear stage of the Pc1 development; connecting wave amplitude, duration of its excitation and dynamics and dynamic of spectrum with plasma parameters. They have shown that in the quasilinear stage, the broadening of the spectrum can take place. A beginning of nonlinear stage can be identified as a moment when the spectrum width begins to grow. During approximately $20 \mathrm{~min}$, the spectrum width became three times larger. This effect with rather broad dynamic spectra of the emissions could be seen in the considered event.

The proposed interpretation of "unusual Pc1 geomagnetic pulsations" is possible, but not the only one. We did not take into account, for example, that simultaneous precipitation of protons into the ionosphere can change the quantitative parameters of the ionosphere, which can lead to a change in the frequency of transmission of the frequency range through the ionospheric Alfven resonator over time (Mursula at al., 2000; Yahnin at al., 2007).

\section{Conclusions}

1. During the late recovery phase of the strong magnetic storm on 7-8 September 2017, it was found the unusual Pc1 pulsation event with a very complicated dynamic spectrum.

2. This Pc1 pulsation event demonstrated the similar frequencytime variations at all 5 ground-based Finnish pulsation magnetometer stations, spaced from $L=3.3$ to $L=5.8$, with the amplitude maximum at the lowest latitude station (NUR,

$$
L=3.3 \text { ). }
$$

3. We showed that the behavior of these unusual Pc1 pulsations was controlled by the solar wind dynamic pressure, the plasmapause location and nonlinear processes in the ambient magnetospheric plasma.

\section{Acknowledgements.}

The work was partly supported by the Project of the Ministry of education and Science KP19-270 "Questions of origin and evolution of the Universe with the use of methods of ground observations and space research" and the state task of IPE RAS. The Pc1 data were obtained from the Sodankylä observatory site (http://WWW.sgo.fi), the solar wind and IMF parameters were taken from the OMNI database (http://omniweb.gsfc.nasa.gov). 


\section{References}

Carpenter, D. L. and Anderson R. R.: 1992, J. Geophys. Res., 97, 1097.

Demekhov, A.G. : 2007, J. Atmos. Solar-Terr. Phys. 69, 1609.

Feygin, F.Z., and Yakimenko, V.L.: 1969, Geomagnetism and Aeronomy. 9(4), 700.

Feygin, F.Z. and Yakimenko, V.L.: 1971, Annales Geophysicae. 27, 49.

Feygin, F.Z., and Kurchashov, Yu.P.: 1975, J.Geomag. Geoelectr. 26, 539.

Feygin, F.Z., Prikner, K., Nekrasov, A.K.: 2003, Geomagnetism and Aeronomy. 43(6), 701.

Gendrin, R., Lacourly, S., Roux, A., Solomon, J., Feygin, F.Z., Gokhberg, M.B., Troitskaya, V.A., and Yakimenko, V.L.: 1971, Planet. Space Sci. 19, 165.

Guglielmi, A., A. Potapov, E. Matveyeva, T. Polyushkina J. Kangas 2006, Adv. Space Res., 38, 1572.

Kangas, J., Guglielmi, A. and Pokhotelov, O.: 1998, Space Sci. Rev. 83, 435.

Matveeva, E.T. and Troitskaya, V.A.: 1965, Geomagnetism and Aeronomy. 5 (6), 1078.

Moldwin, M. B., L. Downward, H. K. Rassoul, R. Amin, and .Anderson,R. R.: 2002, J. Geophys. Res. 107(A11), 1339, doi:10.1029/20022001JA009211.

Mursula, K. J., Prikner, K., Feygin, F.Z., Braysy, T., Kangas, J. Kerttula, R., Pollari P., Pikkarainen, T., and Pokhotelov, O.A.: : 2000, Atmos. Solar-Terr. Phys. 62, 299.

Mursula, K. J. : 2007, Atmos. Solar-Terr. Phys. 69, 1623.

Troitskaya, V.A. and Guglielmi, A.V.: 1969, Russian Physics Uspehi. 97, 453.

Troitskaya, V.A., Baransky, L.N., Matveeva, E.T., Feygin, F.Z., Glangeaud, F., Vinogradov, P.A., Koshelevsky, V.K. : 1975, Geomagnetism and Aeronomy. 15, 524.

Wentworth, R.C. : 1964, J. Geophys. Res., 69, 2291.

Yahnin A.G. and Yahnina T.A.: 2007, J. Atmos. Sol.-Terr. Phys. 69, 1690. 